\title{
20 Divertikelkrankheit des Kolon
}

\author{
Thomas H. K. Schiedeck
}

\subsection{Kapitelzusammenfassung}

Divertikel sind mit einer Prävalenz von ca. $45 \%$ in der Gesamtbevölkerung relativ häufig. Die alleinige Divertikulose hat keinen Krankheitswert. Die Entzündung der Divertikel hingegen führt zu Beschwerden in unterschiedlicher Ausprägung bis hin zu einer Abszessbildung oder Perforation mit Peritonitis und Sepsis. Spätfolgen können Fistelbildung und Stenose sein. Die Therapie ist adaptiert an den Schweregrad der Entzündung bzw. Beschwerden und kann in der Mehrzahl der Fälle konservativ durchgeführt werden. Die operative Therapie unter elektiven Bedingungen wird bevorzugt laparoskopisch durchgeführt. Notfalleingriffe müssen in erster Linie an den Allgemeinzustand des Patienten angepasst werden. Eine Stomaanlage ist dann häufig notwendig.

\subsection{Definition}

Unter dem Begriff „Divertikel“ versteht man erworbene Vorwölbungen von Mukosa und Submukosa durch präformierte Schwachstellen (Muskellücken) in der Kolonwand. Diese Loci minoris resistentiae werden durch schräg angeordnete, intramurale Bindegewebssepten gebildet. In diesen Septen entlang der Tänienränder erreichen die Blutgefäße (Vasa recta) die Kolonmukosa in ihrer gesamten Zirkumferenz (siehe auch Kap. 1.2 Anatomie, Band 1).

Entsprechend dieser Definition handelt es sich bei den typischen Kolondivertikeln um Pseudodivertikel, da sie nicht von allen Wandschichten einschließlich der Muskularis, sondern nur durch Mukosa und Submukosa gebildet werden.

Das Vorhandensein der Kolondivertikel (Divertikulose) muss von dem Begriff der „Divertikulitis“ differenziert werden. Eine Divertikulitis setzt einen Entzündungsprozess voraus, der von Kolondivertikeln ausgeht (Peridivertikulitis), von dort auf die gesamte Darmwand, aber auch auf die Umgebung oder auch benachbarte Organe übergreifen und dadurch klinischen Beschwerden (Abszesse, Perforationen, Fisteln, Stenosen) verursachen kann. 


\subsection{Auftreten}

Die Prävalenz der Divertikulose liegt zwischen $28-45 \%$ in der Gesamtbevölkerung und erreicht über $60 \%$ bei den über 70 -Jährigen.

In den westlichen Ländern sind Kolondivertikel gehäuft im Linkskolon und dort wiederum betont im Sigma zu finden. In Asien hingegen treten Kolondivertikel gehäuft im Rechtskolon auf.

Die Inzidenz der Divertikelkrankheit zeigt eine klare Altersabhängigkeit, wobei letzte Analysen eine Verschiebung zu jüngeren Patienten beobachtet haben.

Unter einer immunsuppressiven Therapie bzw. nach einer Organtransplantation wird ein schwererer Verlauf beschrieben. Im Falle einer komplizierten Verlaufsform der Divertikulitis (z. B. Perforation) liegt die Letalität in der Folge einer Notfalloperation bei $25 \%$.

Bei jüngeren Patienten ist das Risiko eines akuten Entzündungsschubes kumulativ aufgrund der längeren Lebensdauer höher. Daten aus USA zeigen, dass insbesondere bei der Altersgruppe unter 45 Jahren die Hospitalisierung aufgrund einer Divertikulitis zugenommen hat.

\subsection{Pathogenese}

In der Umgebung der Kolondivertikel, insbesondere im Bereich des sogenannten Divertikelhalses, werden gehäuft entzündliche Infiltrationen beobachtet. Histopathologisch werden eine Kryptitis, Ulzerationen mit lymphozytären und neutrophilen Infiltrationen, eine Fibrosierung der Lamina propria mucosae sowie Hyperplasie und Fragmentierung der Lamina muscularis mucosae beschrieben.

Dies kann einerseits über bakterielle Translokation durch die sehr dünne Divertikelwand (keine Lamina muscularis) hervorgerufen werden. Andererseits spielt auch die mechanische Irritation der dünnen Wandung mit konsekutiv möglichen Mikrooder Makroperforationen durch Kotsteine (eingedickter Stuhl) eine Rolle. Hinzu kommt eine reduzierte Durchblutung der Divertikelmukosa durch Kompression der Vase recta im Bereich des Divertikelhalses. Die erwähnten mechanischen Belastungen können auch zu einer direkten Arrosion der Vase recta und damit zur Divertikelblutung führen.

Greift der anfänglich lokale Entzündungsprozess auf benachbarte Organe über, können hieraus Fistelbildungen (bevorzugt in Blase und andere Darmabschnitte, aber auch in die Vagina) resultieren. Wiederholte Entzündungsschübe heilen unter Fibrosierung und Narbenbildung ab. Hieraus entstehen dann im weiteren Verlauf Stenosen und Konglomerattumore (eventuell auch zusammen mit simultaner Fistelung). In Folge der lokalen Entzündung mit dadurch bedingter myostatischer Kontraktur der glatten Darmmuskulatur bzw. auch aufgrund möglicher Stenosierung wird der 
Stuhltransport verlangsamt. Es erhöht sich der intramurale Druck in den proximalen Darmschnitten und die Divertikelbildung schreitet fort.

Die oben erwähnte myostatische Kontraktur wird dabei eher weniger durch eine reine Muskelverdickung, als vielmehr durch eine Hypertrophie der kontrakten Myozyten erzeugt.

Alle derzeitigen Erkenntnisse zeigen einen klaren Zusammenhang im Auftreten der Divertikelkrankheit, aber auch der Divertikulose, und zunehmendem Lebensalter.

Dementsprechend existieren Hinweise, dass zur Divertikelgenese einerseits Alterungsprozesse (Erschlaffung des Bindegewebes und Reduktion des Gewebeturgors mit zunehmendem Lebensalter) beitragen. Darüber hinaus können aber auch anlagebedingte Faktoren eine Rolle spielen. So wurde unter anderem eine Reduktion des Kollagen-Typ I zugunsten des Kollagen Typ III beschrieben. Daneben wurde auch über einen veränderten Enzymbesatz (Metalloproteinasen) und erhöhten Elastingehalt berichtet. Ein indirekter Hinweis für diese Zusammenhänge bildet die Beobachtung, dass insbesondere bei Patienten mit systemischen Bindegewebserkrankungen (wie zum Beispiel Marfan-, Ehlers-Danlos- oder auch Coffin-Lowry-Syndrom) gehäuft Kolondivertikel auftreten.

Einer Zwillingsstudie zufolge zeigt sich ein klares genetisches Risiko für eine Divertikelentwicklung mit einer Odds-Ratio von 7,15 für den monozygoten Zwilling. Nach diesen Zahlen schätzt man den Einfluss genetischer Faktoren auf eine Divertikelerkrankung auf immerhin $40 \%$ (im Vergleich zu $60 \%$ für Umweltfaktoren).

Forschungsergebnisse weisen auch daraufhin, dass bei der Divertikelkrankheit eine Neuropathie im enterischen Nervensystem, verursacht durch strukturelle Veränderungen, aber auch durch Störungen im Neurotransmittersystem, vorliegen können. Bedeutsam erscheint auch, dass ein wahrscheinlich postinflammatorisch hervorgerufener Anstieg von schmerzvermittelnden Neurotransmittern sowie eine Proliferation von schmerzleitenden Afferenzen schließlich eine viszerale Hypersensitivität (insbesondere bei chronifizierten Verlaufsformen) hervorrufen kann. Ähnliches wird beim postinfektiösen Reizdarmsyndrom beobachtet. Einige Untersuchungen weisen auch daraufhin, dass bei Divertikelträgern im Rektum eine erhöhte sensorische Empfindlichkeit sogar im divertikelfreien Rektum vorliegen kann.

\subsection{Einteilung}

Weltweit und insbesondere in den angloamerikanischen Ländern wird die Divertikulitis nach Hinchey eingeteilt. Diese Form der Klassifikation ist jedoch relativ unpräzise, da sie nur auf intraoperative Befunde zurückgreift bzw. Befunde der Bildgebung daraufhin extrapoliert. Basierend auf den gemeinsamen Leitlinien von DGVS und DGAV ist folgende Klassifikation der Divertikulitis (CDD) zu bevorzugen, siehe hierzu auch Tab. 20.1: 
Tab. 20.1: Klassifikation der Divertikelerkrankung nach den Leitlinien der DGVS/DGAV.

\begin{tabular}{|c|c|c|}
\hline Typ 0 & Asymptomatische Divertikulose & Zufallsbefund; asymptomatisch \\
\hline Typ 1 & Unkomplizierte Divertikulitis & $\begin{array}{l}\text { divertikelbezogene Symptome; Entzün- } \\
\text { dungszeichen, Bildgebung mit spezifischem } \\
\text { Befund }\end{array}$ \\
\hline Typ 1a & $\begin{array}{l}\text { Divertikulitis ohne Umgebungs- } \\
\text { reaktion }\end{array}$ & $\begin{array}{l}\text { Entzündungszeichen (Labor, Klinik), ent- } \\
\text { zündetes Divertikel }\end{array}$ \\
\hline Typ 1b & $\begin{array}{l}\text { Divertikulitis mit phlegmonöser } \\
\text { Umgebungsreaktion }\end{array}$ & $\begin{array}{l}\text { Phlegmone Divertikulitis mit Wandver- } \\
\text { dickung, Peridivertikulitis }\end{array}$ \\
\hline Typ 2 & akute komplizierte Divertikulitis & \\
\hline Typ 2a & Mikroabszess & $\begin{array}{l}\text { gedeckte Perforation, kleiner Abszess } \\
(\leq 1 \mathrm{~cm} \text {; minimale parabolische Luft) }\end{array}$ \\
\hline Typ 2b & Makroabszess & parakolischer oder mesokolischer Abszess \\
\hline Typ 2c & freie Perforation & freie Perforation, freie Luft/Flüssigkeit \\
\hline Typ 2c1 & eitrige Peritonitis & generalisierte Peritonitis \\
\hline Typ 2c2 & fäkale Peritonitis & \\
\hline Typ 3 & chronische Divertikelkrankheit & $\begin{array}{l}\text { rezidivierende oder anhaltend symptomati- } \\
\text { sche Divertikelkrankheit }\end{array}$ \\
\hline Typ 3a & $\begin{array}{l}\text { symptomatische unkomplizierte Di- } \\
\text { vertikelkrankheit (=SUDD) }\end{array}$ & $\begin{array}{l}\text { DD Reizdarm; Entzündungszeichen optional } \\
\text { (Divertikulosenachweis) }\end{array}$ \\
\hline Typ 3b & $\begin{array}{l}\text { rezidivierende Divertikulitis ohne } \\
\text { Komplikationen }\end{array}$ & Entzündungszeichen, entzündetes Divertikel \\
\hline Typ 3c & $\begin{array}{l}\text { rezidivierende Divertikulitis mit Kom- } \\
\text { plikationen }\end{array}$ & Fistel, Stenose, Konglomerat \\
\hline Typ 4 & Divertikelblutung & Blutungsquelle/Divertikel \\
\hline
\end{tabular}

DD Differenzialdiagnose, DGAV Deutsche Gesellschaft für Allgemein- und Viszeralchirurgie, DGVS Deutsche Gesellschaft für Verdauungs- und Stoffwechselkrankheiten

Eine Divertikelkrankheit des Kolons liegt vor, wenn eine Divertikulose zu Symptomen oder Komplikationen führt. Als symptomatische unkomplizierte Divertikelkrankheit werden persistierende oder rezidivierende Divertikel-assoziierte Symptome ohne begleitende, klinisch manifeste Entzündungszeichen beschrieben.

Von einer akuten Divertikulitis hingegen spricht man, wenn sich eine Entzündung der Pseudodivertikel oder angrenzender Strukturen entwickelt hat. Eine akute, komplizierte Form der Entzündung liegt vor, wenn zusätzlich ein Abszess (möglicherweise mit Fistelbildung) bzw. eine Perforation aufgetreten ist (Abb. 20.1). Die chronische Form der Divertikulitis hingegen ist definiert durch bestehende oder wiederholte Be- 


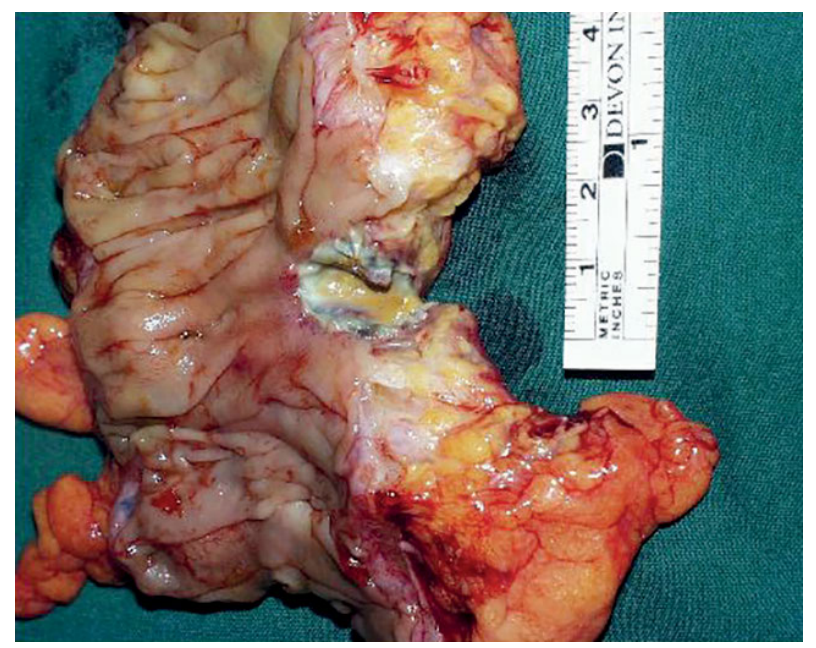

Abb. 20.1: Resektat mit intramuraler Abszedierung und Perforation.

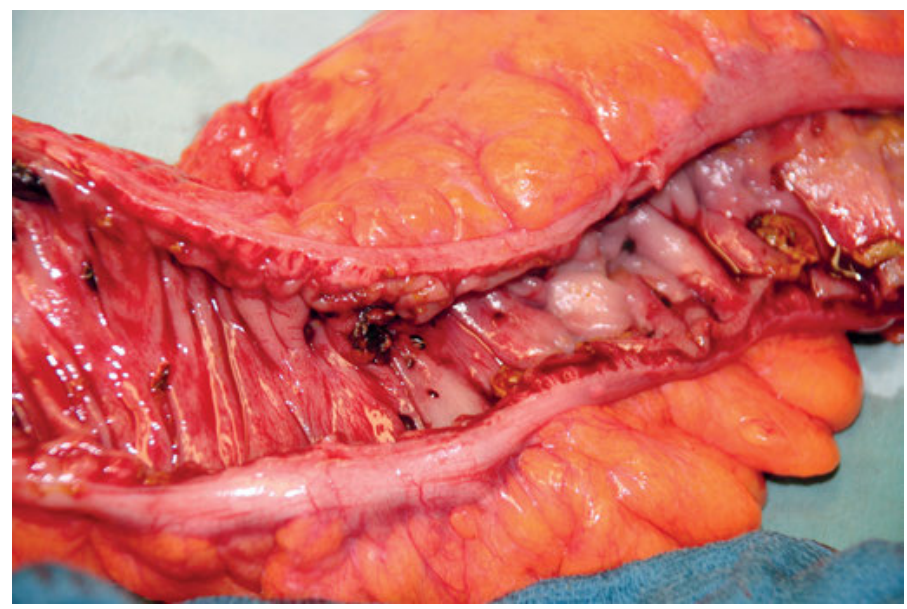

Abb. 20.2: Operationspräparat mit Stenosierung und multiplen Divertikelöffnungen.

schwerdeepisoden - Entzündungszeichen sind nicht immer vorhanden. Auch Spätkomplikationen (Fisteln, Stenosen) sind bekannt (Abb. 20.2). Gerade bei der chronisch rezidivierenden unkomplizierten Verlaufsform ist die Differentialdiagnose zum Reizdarm mitunter sehr schwierig zu stellen.

Eine Divertikulitis tritt zwar bevorzugt im Linkskolon auf, kann sich jedoch durchaus auch im Kolon ascendens und Coecum manifestieren. Nicht selten wird die Symptomatik dann als Appendizitis fehlinterpretiert. Wie oben bereits erwähnt ist eine Divertikelblutung ursächlich auf eine Ruptur der transmural verlaufenden Vasa recta zurückzuführen. Klinisch manifestiert sie sich als transanaler Blutabgang, welcher in schweren Fällen sehr massiv auftreten kann und dann auch kreislaufwirksam wird. 


\subsection{Prophylaxe und Verlauf}

Zur Primärprophylaxe einer Divertikulitis werden derzeit regelmäßige körperliche Aktivität, ballaststoffreiche Ernährung und vegetarische Kost empfohlen. Normalgewicht ist ein zusätzlich günstiger Faktor.

Die Daten über den Verlauf bzw. das Rezidivverhalten einer stationär therapierten Divertikulitis sind sehr breit gestreut. Bei einer ursprünglich unkomplizierten Divertikulitis wird in ca. 2\%, bei Patienten mit schweren Verläufen hingegen in 35\% der Fälle mit einem Rezidiv zu rechnen sein. Je nach Ausprägung und Häufigkeit der Symptomatik kann die Lebensqualität nach einer akuten Divertikulitis reduziert sein.

\subsection{Diagnostik}

\subsubsection{Anamnese}

Die Patienten beschreiben in erster Linie Schmerzen, teils stechend teils schneidend, bevorzugt (aber nicht ausschließlich) im linken unteren Quadranten. Diese Beschwerden sind häufig durch Meteorismus/Flatulenz begleitet. Nach dem Stuhlgang bzw. nach Luftabgang wird Besserung berichtet.

Bei Frauen sollte eine gynäkologische Ursache der Beschwerden bedacht und dementsprechend eine fachärztliche Abklärung veranlasst werden.

Wechselndes Stuhlverhalten (Obstipation - Diarrhoe) kann auf eine Stenosierung hinweisen. Treten Harnentleerungsbeschwerden hinzu oder wird über Pneumaturie, Hämaturie oder auch Luftabgang per vaginam berichtet, kann dies auf komplizierende Verlaufsformen (Stenose, Fistel, Abszess) hinweisen. Differentialdiagnostisch muss dann auch an eine Tumorerkrankung gedacht werden.

Wichtig: Auch bei jüngeren Patienten und auch bei untypischer Schmerzlokalisation (rechter Unterbauch, suprapubisch) sollte an eine Divertikulitis als mögliche Ursache der Beschwerden gedacht werden (Variable Lage des Colon sigmoideum, Divertikulitis des Colon ascendens, gedeckter Abszess).

\subsubsection{Palpation/Auskultation}

Typischerweise kann man im Stadium der akuten Divertikulitis im linken Unterbauch eine druckdolente Walze tasten. Im chronischen Stadium ist dies dann deutlich diskreter. Im Falle einer Perforation hingegen werden die klinisch palpatorischen Signale einer Peritonitis manifest: Lokale oder auch diffuse Abwehrspannung, Klopfschmerz, Schonhaltung der Beine. Auskultatorisch lassen sich je nach klinischer Situation entweder hochgestellte Darmgeräusche als Zeichen eines beginnenden Ileus finden, 
plätschernde Stenosegeräusche sind ebenso möglich, wie die „Totenstille“ im Vollbild des Ileus bei einer Peritonitis (Darmparalyse).

\subsubsection{Labor}

Zu den Kennzeichen einer Divertikulitis (im Gegensatz zur Divertikulose) gehört eine Erhöhung der Entzündungsparameter: Temperaturerhöhung (>37,6-38 Grad Celsius), Leukozytose (>10-12.000 $\mu$ l), Anstieg des C-reaktiven Proteins (CRP) im Serum ( $>5 \mathrm{mg} / 100 \mathrm{ml}$ ). Um einen Harnwegsinfekt (z. B. im Rahmen einer Fistel) zu erfassen, sollte der Urinstatus überprüft werden.

Am verlässlichsten erscheint das CRP geeignet im klinischen Kontext eine Divertikulitis zu objektivieren. Die Höhe des CRP korreliert dabei tendenziell mit komplizierten/perforierten Verläufen. Dabei spiegeln Werte $>5 \mathrm{mg} / 100 \mathrm{ml}$ eine Divertikulitis wieder, während ein CRP $>20 \mathrm{mg} / 100 \mathrm{ml}$ den Verdacht auf eine Perforation erweckt. Allerdings ist der negative prädiktive Wert sowohl des Blutbildes (Leukozyten) als auch des CRP relativ schlecht, sodass auch bei Normalwerten eine Entzündung nicht ausgeschlossen werden kann.

Die beschriebenen Entzündungszeichen entwickeln sich oft erst im weiteren Krankheitsverlauf, d.h. in der Regel innerhalb von 1-2Tagen, daher ist in diesem Zeitfenster auch eine Kontrolle der Parameter (zusammen mit der klinischen Untersuchung) absolut indiziert.

\subsubsection{Bildgebung}

Die alleinige klinische Untersuchung hat zur Abklärung einer Divertikulitis lediglich eine Sensitivität von ca. 70 \%, der positive prädikative Wert ist mit 65-67\% ähnlich unbefriedigend.

Dementsprechend kommt der Bildgebung im Rahmen der Diagnostik und Differentialdiagnostik einer Divertikulitis entscheidende Bedeutung zu.

Viele Studien belegen, dass in diesem Zusammenhang Sonographie (Abb. 20.3) und Computertomographie (CT) mit intravenöser (und intestinaler) Kontrastierung gleichwertige Schnittbildverfahren sind. Beide Techniken erfordern nicht nur eine adäquate und zeitgemäße Ausstattung, sondern eben auch große Expertise des Untersuchers. Da die Sonographie zudem ohne Strahlenbelastung anzuwenden ist, stellt sie an sich das bevorzugte Untersuchungsinstrument dar. Internationale Daten zeigen allerdings, dass auf dem Gebiet der Sonographie eine flächendeckende Diagnostik in der geforderten Qualität rund um die Uhr nicht in gleichem Maße gegeben ist und daher wird weltweit die CT des Abdomens bevorzugt empfohlen. Es ist allerdings auch bekannt, dass insbesondere bei frühen Divertikulitisstadien (Phlegmone, Peridivertikulitis) die CT zu einem signifikanten Overstaging neigt. 


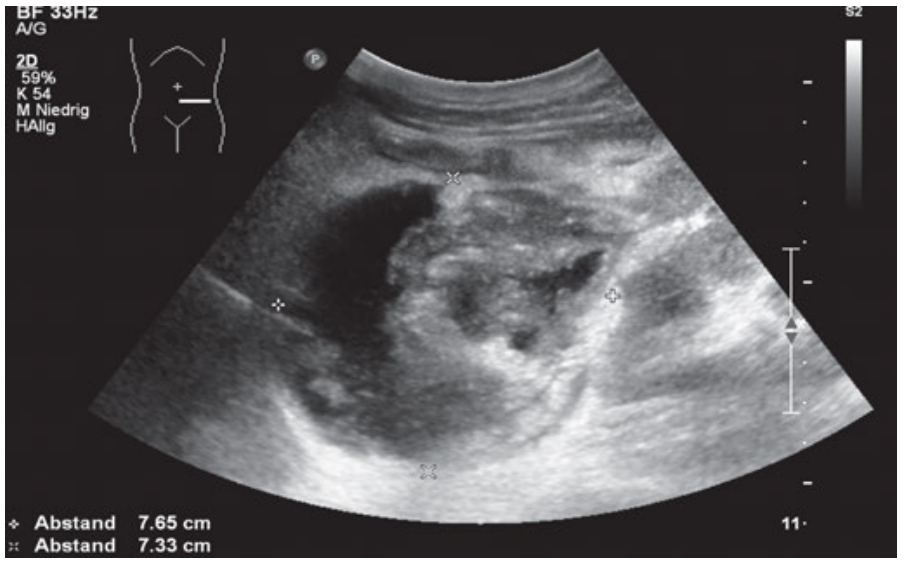

Abb. 20.3: Sonographisches Bild einer perforierten Divertikulitis mit Abszessbildung, Punktion bei 9:00.

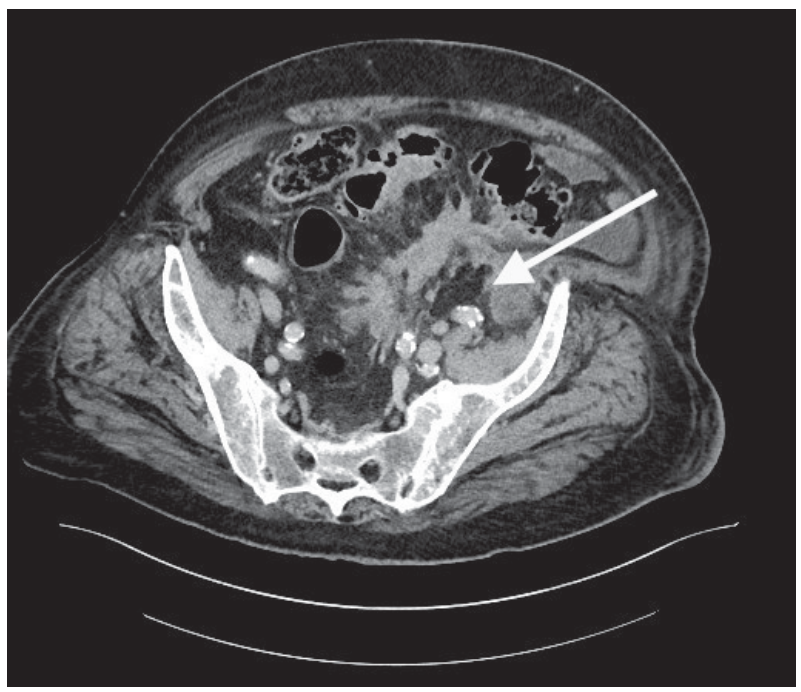

Abb. 20.4: Sigmadivertikulitis mit intramesenterialer bzw. retroperitonealer Abszedierung (Pfeil).

Besonders in der Diagnostik tiefer mesenterial bzw. im kleinen Becken gelegener Abszessformationen (Abb. 20.4) ist die Computertomographie der Sonographie überlegen. Die CT hat zudem den Vorteil, die Diagnostik direkt mit therapeutischen Verfahren verbinden zu können (Punktion von freier intraabdomineller Flüssigkeit, Platzierung auch tiefer liegender Abszessdrainagen). Eine CT-Diagnostik liefert wichtige Informationen bei der Selektion eines eventuell notwendigen operativen Verfahrens (Zugangswege, Trokarplatzierung etc.).

In der Primärdiagnostik einer akuten Divertikulitis wird in Deutschland auch aus Gründen des Strahlenschutzes primär die Abdomensonographie eingesetzt. Sollte sich allerdings die Befundkonstellation des Patienten unter Therapie nicht bessern oder gar verschlechtern, sollte in jedem Fall eine CT veranlasst werden. Damit können 
neu entstandene oder eventuell auch primär übersehene Abszessformationen, Fisteln oder Perforationen (= Wechsel in eine komplizierte Divertikulitis) nachgewiesen werden. Dies gilt gleichermaßen vor einer operativen Intervention zur besseren Planung und adäquaten Aufklärung des Patienten bezüglich anstehender Maßnahmen und möglicher Alternativen.

Die Kernspintomographie hat im Rahmen der Divertikulitis keinen diagnostischen Stellenwert, der Kontrasteinlauf ist obsolet.

\subsubsection{Endoskopie/Proktorektoskopie}

Bei einer akuten Divertikulitis ist eine Koloskopie nicht indiziert. Sie ist hingegen Mittel der Wahl zur Abklärung einer intestinalen Blutung oder zum Ausschluss einer (tumorbedingten) Stenosierung. Eine (gedeckte) Perforation sollte vor Durchführung einer endoskopischen Diagnostik in der Notfallsituation ausgeschlossen werden. Vor einer (früh)elektiven operativen Intervention (z. B. Sigmaresektion) hingegen, sollte eine koloskopische Abklärung zum Ausschluss eines operationsrelevanten Zweitbefundes durchgeführt werden.

Die starre Proktorektoskopie wird in erster Linie eingesetzt, um vor einer geplanten Resektion, technische Hindernisse einer Anastomosierung abzuklären.

\subsubsection{Konsiliarische Untersuchungen}

Insbesondere bei Frauen ist eine gynäkologische Vorstellung aus differentialdiagnostischen Überlegungen relevant (s. o.). Der Nachweis einer Sigma-Blasenfistel oder auch einer sigmavaginalen Fistel kann selbst mit Hilfe einer CT-Diagnostik mitunter schwierig sein. Auch eine Zystoskopie durch den urologischen Facharzt ist nicht in allen Fällen wegweisend. Im Zweifel ist daher der Mohnsamen-Test nach wie vor ein probates Mittel, um die Indikation zu einer Operation (z. B. Sigmaresektion) auf eine stabile Basis zu stellen.

Mohnsamen-Test: Pat. erhalten am Abend ca. 35-50 g Mohnsamen in ca. $150 \mathrm{~g}$ Joghurt. Danach wird der Urin (sigmavesikale Fistel) auf Mohn kontrolliert oder zur Abklärung einer sigmavaginalen Fistel z. B. ein Tampon vaginal platziert und dann auf Mohnaustritt kontrolliert. 


\subsection{Therapie}

\subsubsection{Konservativ}

Die akute unkomplizierte Divertikulitis bei Patienten mit Risikofaktoren wird durch eine Antibiotikatherapie behandelt. Bei Patienten ohne Risikofaktoren hingegen kann-unter engmaschiger Verlaufskontrolle-auf eine Antibiotikatherapie verzichtet werden. Die Behandlung kann ambulant durchgeführt werden, wenn keine sekundären Entzündungszeichen wie Fieber oder Leukozytose vorliegen, kein Perforationsverdacht besteht und eine ausreichende Compliance des Patienten eine ausreichende Flüssigkeits- und Nahrungszufuhr ermöglicht.

Als Risikofaktoren für einen komplizierten Verlauf zählt man neben Immunsuppression und chronischen Nierenerkrankungen Patienten mit arterieller Hypertonie und allergischer Disposition.

Patienten mit einer komplizierten Verlaufsform der Divertikulitis werden in der Regel stationär behandelt. Die Therapie besteht in der Regel aus Antibiotikatherapie und zusätzlich, insbesondere bei drohender negativer Bilanz, in parenteraler Flüssigkeitssubstitution. Eine orale Nahrungszufuhr ist je nach Ausprägung der akuten Entzündungsmanifestation möglich. Bewährte Antibiotikakombinationen sind Cefuroxim oder Ciprofloxacin jeweils zusammen mit Metronidazol oder auch Ampicillin/ Sulbactam bzw. Piperacillin/Tazobactam.

Für die akute unkomplizierte Divertikulitis gilt generell: Falls eine adäquate konservative Therapie nicht zur Ausheilung führt, muss nach Abwägen differentialdiagnostischer Alternativen (unter Einsatz geeigneter diagnostischer Mittel-s.o.), die operative Therapie diskutiert werden.

\subsubsection{Operativ}

Eine operative Therapie zur Behandlung einer Divertikulitis muss nach sehr verschiedenen Grundbedingungen differenziert werden:

- Notfall-Operation: Der Eingriff ist sofort indiziert und muss unverzüglich durchgeführt werden.

- Dringliche Operation: Ein Eingriff, der innerhalb eines kurzen Zeitfensters ( $24 \mathrm{~h})$ durchgeführt werden muss. Hier sind noch Maßnahmen zur perioperativen Verbesserung des Patienten bzw. zur diagnostischen Abklärung möglich.

- Frühelektive Operation: Dieses Intervall ist in der Literatur nicht klar definiert. Manche Autoren geben hierfür ein relativ kurzes Empfehlungsintervall (48 h), andere stecken das Fenster deutlich weiter (3-5 Tage), manche definieren damit eine Operation innerhalb desselben stationären Aufenthaltes und verbinden es mit dem Abklingen der akuten Entzündungssymptomatik. 
- Elektive Operation: Der Eingriff ist langfristig zu planen und wird im symptomfreien Intervall durchgeführt.

Die einzige Indikation für eine sofortige Notfalloperation ist der Patient mit den Zeichen einer diffusen Peritonitis bzw. der kreislaufinstabile septische Patient. Im letzteren Fall wird man in der Regel stabilisierende Maßnahmen vorschalten, wobei diese nicht mehr als 2-3h in Anspruch nehmen sollten. Ansonsten wird man prinzipiell immer anstreben, einen operativen Eingriff erst nach Abklingen der akuten Entzündungssymptomatik durchzuführen. Das heißt, auch bei einer Perforation mit Abszessbildung oder mit Fistelung unter Ausprägung eines Konglomerat-Tumors wird man zunächst mit einer konservativen Therapie beginnen. Diese besteht dann, wie oben bereits ausgeführt, aus parenteraler Ernährung und intravenöser Antibiotikagabe. Sie wird flankiert von abführenden Maßnahmen, wobei hier insbesondere bei Perforationsverdacht Vorsicht geboten ist. Eine forcierte Darmlavage sollte in diesen Fällen nicht durchgeführt werden. Eine systematische Literaturanalyse aus dem Jahr 2016 bei 8.766 Patienten mit einer abszedierten Divertikulitis zeigt, dass Abszesse $<3 \mathrm{~cm}$ sehr gut antibiotisch behandelt werden können, größere Abszesse sollten jedoch bevorzugt über eine perkutane Drainage entlastet werden. Dies kann CT- oder sonographiegesteuert erfolgen. Insgesamt war die primär konservative Therapie in $80 \%$ zunächst erfolgreich. Patienten, die im Bild einer akuten abszedierten Divertikulitis sofort operativ versorgt wurden, hatten immerhin ein Letalitätsrisiko von 12,1\%. Aus diesem Grund sollte der primär konservativen Behandlung, wenn möglich der Vorzug gegeben werden.

\section{Indikation zur Operation}

- Akute unkomplizierte Divertikulitis (Typ 1, siehe Tab.20.1): Die akute unkomplizierte Divertikulitis wird primär konservativ behandelt. Nur wenn trotz adäquater Therapie keine Besserung oder gar eine Verschlechterung $\mathrm{zu}$ verzeichnen ist, stellt sich die Frage einer Operation. Vorher sollten dann Differentialdiagnosen und mögliche neu aufgetretene Komplikationen einer Divertikulitis mit Hilfe einer nochmaligen Schnittbilddiagnostik ausgeschlossen werden. Eine elektive Resektion im Rahmen einer Rezidivprophylaxe ist nur in Ausnahmefällen zu erwägen (s.u.).

- Akute komplizierte Divertikulitis (Typ 2, siehe Tab.20.1): Eine akute komplizierte Divertikulitis ohne generalisierte Peritonitis- oder Sepsiszeichen wird zunächst konservativ behandelt (s. o.). Die Ergebnisse vorliegender Studien sind hier aktuell zwar in ihrer Bewertung nicht unisono und eindeutig, aber unter Berücksichtigung der aktuellen Leitlinie wird eine operative Versorgung mit Resektion im frühelektiven oder elektiven Intervall empfohlen. Histologische Untersuchungen belegen, dass nach gedeckter Perforation gravierende strukturelle Veränderungen der Darmwand dauerhaft nachzuweisen sind. Insbesondere bei Makroabs- 
zessformationen, die drainiert wurden, sollte man einer frühelektiven Resektion im Rahmen desselben stationären Aufenthalts den Vorzug geben.

- Die freie Perforation mit Peritonitis und Sepsis (Typ 2c, siehe Tab. 20.1): Eine freie Perforation mit dem klinischen Bild eines akuten Abdomens, Typ 2c (inkl. c1 und c2) stellt eine Indikation zur sofortigen Notfalloperation dar. Unklar ist, auch unter Berücksichtigung aktueller Studien, nicht die Frage der prinzipiellen Operationsindikation, sondern die Wahl des geeigneten Verfahrens: Hier wird derzeit nicht nur der Zugangsweg, sondern auch das Ausmaß bzw. die Notwendigkeit einer Resektion diskutiert. Zusätzlich haben mehrzeitige Operationsstrategien wieder einen neuen Stellenwert („Bridging“-„Damage Control“) gewonnen. Besondere klinische Herausforderungen bleiben Patienten ohne sicheren radiologischen Nachweis einer Perforation oder Abszedierung aber mit generalisierter Peritonitis. Nach dem differentialdiagnostischen Ausschluss anderer Erkrankungsursachen (z. B. Pyelonephritis, Colitis), wird man sich zu einer operativen Exploration des Abdomens in Verbindung mit einer Lavage entschließen.

- Chronisch rezidivierende Divertikulitis (Typ 3, siehe Tab.20.1): Die symptomatische, unkomplizierte Divertikelkrankheit (SUDD, Typ 3a, s. o.) und die unkomplizierte rezidivierende Divertikulitis (3b) werden primär konservativ behandelt. Eine prinzipielle Operationsempfehlung aus Gründen der Rezidivprophylaxe kann nicht ausgesprochen werden. Darüber hinaus hat sich gezeigt, dass auch wiederholte Entzündungsschübe nicht mit einem erhöhten Perforationsrisiko einhergehen. Daher spricht das Ergebnis vieler Beobachtungsstudien für eine wiederholte konservative Therapie. Es gibt allerdings histomorphologische Hinweise, die zeigen, dass selbst die SUDD klinisch nicht-manifeste, aber histologisch erkennbare entzündliche Veränderungen besitzt. Neuro-peptiderge Veränderungen in der Schleimhaut erklären dann auch hartnäckige schmerzhafte Beschwerden dieser Patienten. Diese Beobachtungen und neuere Studien zur Lebensqualität zeigen, dass diese Patientengruppen daher auch von einer operativen Therapie profitieren können. Die Entscheidung zu einer Operation ist dann immer eine individuelle Abwägung, die nicht allein die Anzahl möglicher Schübe berücksichtigt, sondern darüber hinaus die persönliche Lebenssituation des Patienten, die Häufigkeit des Auftretens, die Schmerzintensität und notwendige Behandlungsdauer bewerten sollte. Der DIRECT-Trial hat immerhin gezeigt, dass operativ behandelte Patienten aus dieser chronischen Verlaufsgruppe selbst nach einer postoperativen Komplikation, eine bessere Lebensqualität erreichten, als konservativ Behandelte.

Problematisch bleibt die Tatsache, dass insbesondere in der Gruppe Typ 3a eine Abgrenzung zum Reizdarmsyndrom nicht möglich ist. Da der Reizdarm wiederum operativ nicht positiv beeinflusst werden kann, besteht hier das nicht unerhebliche Risiko der Beschwerdepersistenz oder gar Verschlechterung. Dies muss im Rahmen eines 
präoperativen Aufklärungsgesprächs speziell mit den Patienten abgewogen und partizipativ entschieden werden.

Patienten mit einem komplizierten chronisch rezidivierenden Verlauf $(3 c=$ Stenose, Fistel, siehe Tab. 20.1) sollten operiert werden. Jede Fistel zum Harntrakt birgt ein hohes Risiko der Urosepsis und stellt daher eine prinzipielle Operationsindikation dar. Fisteln zur Vagina stellen in erster Linie eine massive Einschränkung der Lebensqualität für die Patientin dar, da der permanente unkontrollierbare Stuhlabgang nicht nur lokale Entzündungen hervorruft, sondern auch nicht soziale Isolation bedeutet. Wenn den Patienten aufgrund eines generell schlechten Allgemeinzustandes eine resezierende (multiviszerale) Operation nicht zugemutet werden kann, sollte als Minimalmaßnahme ein Kolostoma zur Deviation angelegt werden.

Stenosen sind dann operationspflichtig, wenn aus ihnen eine relevante Störung der Stuhlpassage resultiert.

\section{Risikogruppen}

Immunsupprimierte Patienten bzw. Organtransplantierte, chronisch Nierenkranke, aber auch Patienten im fortgeschrittenen ASA Stadium (III) gelten als Patienten mit einem erhöhten Risiko. Deswegen sollte hier nach einem stationär behandlungsbedürftigen Divertikulitisschub über eine elektive Resektion diskutiert werden. Dabei ist das Rezidivrisiko nicht häufiger, aber prinzipiell ist das mögliche Rezidiv schwieriger medikamentös zu behandeln und eine eventuelle Notfalloperation mit einer doppelt so hohen Mortalität (> 25\%) als bei einem Normalkollektiv verbunden.

\section{Tumorverdacht}

Besteht präoperativ oder intraoperativ der Verdacht eines malignen Tumors - eventuell mit begleitender oder komplizierender Divertikulitis - so ist eine tumortaktische Vorgehensweise unter Beachtung onkologischer Radikalitätsprinzipien indiziert. Im Zweifel kann auch ein mehrzeitiges Vorgehen unter Anlage eines deviierenden Stomas gewählt werden.

\section{Blutung}

Die schmerzlos auftretende untere gastrointestinale Blutung ist in ca. 35\% der Fälle eine Divertikel-assoziierte Blutung. Diese ist in der Mehrzahl der Fälle selbstlimitierend. Sie ist eher die Folge einer langjährig bestehenden Divertikelerkrankung als Ausdruck einer akuten Divertikulitis. Das Blutungsereignis an sich stellt dann auch keine Operationsindikation dar. Das Risiko einer erneuten Blutung muss in einem Intervall von 4 Jahren in 13,8 \% der Fälle erwartet werden, wobei auch diese Blutungen in der Regel von alleine sistieren. Anhand des Transfusionsbedarfs kann grob abgeschätzt werden, wie hoch die Chance ist, dass eine Divertikelblutung spontan sistiert: Beträgt der Transfusionsbedarf 1-2 Erythrozytenkonzentrate (EKs) pro Tag, 
sistieren einer Studie zufolge nahezu $100 \%$ der Blutungen, bei 3 EKs waren es noch $88 \%$. Doch schon bei $5 \mathrm{EKs} / \mathrm{Tag}$ ist damit nur noch in $43 \% \mathrm{zu}$ rechnen und bei einem Verbrauch von 6 oder mehr EKs fällt die Rate auf $0 \%$.

Wie bei jeder gastrointestinalen Blutung sollte zunächst eine endoskopische Lokalisationsdiagnostik angestrebt werden. Gegebenenfalls kann dann direkt im Anschluss daran die Blutung interventionell gestillt werden (Clip, Unterspritzung). Idealerweise wird der Darm hierfür durch eine orthograde Darmspülung vorbereitet und die Koloskopie dann innerhalb 12-24 h angeschlossen.

Bei wiederholter oder anhaltender Blutung ohne endoskopisch lokalisierbare Blutungsquelle empfiehlt sich die Durchführung einer CT-Angiographie. Diese hat einerseits das Ziel der Lokalisation und bietet zusätzlich die Option einer Blutungsstillung durch superselektive Embolisation. Selbst wenn die Blutungsquelle damit nicht kontrolliert werden kann, liefert ihre Lokalisation für eine nachfolgende Notfalloperation doch wichtige Informationen damit das Resektionsausmaß limitiert werden kann.

Sollte eine präoperative Lokalisationsdiagnostik scheitern oder aufgrund schlechter Kreislaufverhältnisse nicht mehr möglich sein, bleibt in diesen Fällen die subtotale Kolektomie - gegebenenfalls mit endständiger Ileostomie - das Verfahren der Wahl.

\section{Resektionsausmaß und Verfahrenswahl}

Da im häufigsten Fall bei Patienten der westlichen Industrienationen eine Sigmadivertikulitis vorliegt, ist die Sigmaresektion der typische Eingriff. In Abhängigkeit des Entzündungsausmaßes und der anatomischen Gegebenheiten wird er in manchen Fällen im Sinne einer Hemikolektomie links nach proximal zu erweitern sein. In den wenigen Fällen einer rechtsseitigen Divertikulitis ist natürlich die rechtsseitige Resektion (Hemikolektomie rechts - gegebenenfalls unter Mitnahme des proximalen Kolons transversums) indiziert.

Die Divertikulitis ist eine benigne Erkrankung, daher ist ein operatives Verfahren gemäß onkologischer Radikalitätskriterien nicht indiziert. Dementsprechend bleibt die radikuläre mesenteriale Gefäßversorgung intakt, die Resektionslinie verläuft idealerweise darmwandnah. Dadurch sind die Durchblutungsverhältnisse im Anastomosenbereich optimal und das Risiko einer Nahtinsuffizienz wird reduziert. Zudem bleibt bei dieser Vorgehensweise auch der präaortal liegende vegetative Nervenplexus geschont. Bei jeder Linkskolondivertikulitis sollte das gesamte Sigma reseziert werden. Die distale Resektionslinie liegt damit im oberen Rektumdrittel. Dieses ist klinisch daran zu erkennen, dass dort keine Taenien und keine Appendices epiploicae mehr vorhanden sind. Dies muss konsequent beachtet werden, da der größte Risikofaktor für ein Divertikulitisrezidiv eine inadäquate aborale Absetzungsgrenze darstellt. Unter optimalen Bedingungen hingegen muss in lediglich $6 \%$ mit einem Divertikulitisrezidiv gerechnet werden (nach konservativer Therapie beträgt das Ri- 
siko eines Rezidivs hingegen $18 \%$ ). Die proximale Resektionsgrenze liegt zirka eine Handbreit im entzündungsfreien Kolon. Es ist dabei keineswegs das Ziel alle divertikeltragenden Darmanteile zu entfernen, sondern lediglich das entzündete Segment. Allerdings sollte der direkte Anastomosenbereich frei von Divertikeln sein.

\section{Elektive Resektion}

Die bevorzugte und empfohlene Operation der Sigmadivertikulitis ist die Sigmaresektion. Hierbei ist die laparoskopische Operationstechnik der offenen überlegen - es sei denn, wichtige klinische Gründe (Verwachsungen, anatomische Besonderheiten, anästhesiologische Faktoren) oder auch der Wunsch des Patienten sprechen dagegen.

Nach der Literatur ist es aktuell wiederum als gleichwertig zu betrachten, ob die Resektion laparoskopisch in typischer Mehrtrokartechnik oder in sogenannter „Single-Incision“-Methode bzw. robotisch-assistiert oder auch in transanal minimal invasiver (TAMIS) Weise erfolgt. Die klassische laparoskopische Technik ist die am weitesten verbreitete. Alle anderen Verfahren haben ihre technische Machbarkeit und Sicherheit erwiesen, sind ihr jedoch bislang nicht signifikant überlegen.

\section{Resektion im Notfall}

Vielen Fallserien bzw. Fallkontrollberichte belegen inzwischen, dass auch in der Notfallsituation ein laparoskopisches Vorgehen möglich ist und in erfahrenen Händen auch Vorteile für den Patienten bieten kann.

Allerdings ist gerade im Notfall die Varianz unterschiedlicher klinischer Szenarien sehr breit gefächert, so dass in diesen Fällen die Verfahrenswahl multiplen Einflussgrößen unterworfen ist. In erster Linie ist hier sicher der Allgemeinzustand des Patienten (Adipositas und andere Komorbiditäten bzw. Risikofaktoren s. o.), sein Lebensalter und die Kreislaufstabilität zu beachten. Bei einem hochseptischen älteren Patienten, der auch für die Narkoseführung eine erhebliche Anforderung stellt, wird man das primär offene Verfahren bevorzugen, da hier eine Herdsanierung am schnellsten zu erreichen ist.

So variantenreich die mögliche Notfallsituation, so unterschiedlich sind inzwischen die dafür vorgeschlagenen möglichen Operationsverfahren:

Es ist eine viele Jahre währende Diskussion inwieweit in der Akutsituation eine sichere primäre Anastomose angelegt werden kann und wann eine Diskontinuitätsresektion favorisiert werden sollte. Auch dies wird zweifelsohne immer von der klinischen Situation abhängen und vom jeweiligen Operateur, basierend auf seiner persönlichen Expertise beurteilt werden müssen. Aber es ist ein klarer Fakt, der in vielen Studien belegt werden konnte, dass auch in der Notfallsituation die Anlage einer primären Anastomose gut und sicher möglich ist. In der Regel wird dann eine protektive Ileostomie davor geschaltet. Es ist außerdem belegt, dass nach einer Diskontinuitätsresektion nach Hartmann mit endständiger Kolostomie viele Patienten (55\%) letztlich keine Wiederherstellung der Darmpassage erfahren und dass zu- 
sätzlich die Reanastomosierung auch mit signifikanter Komorbidität (bis 30\%) und Letalität ( $5 \%$ ) verbunden ist.

In diesem Dilemma bietet der Ansatz „Damage control“, welcher ursprünglich aus der Militärchirurgie stammt, eine sehr gute zusätzliche Option. Bei dieser Technik beschränkt man sich im primären Notfalleingriff ausschließlich auf die Herdsanierung (Resektion des entzündeten bzw. perforierten Segmentes, Lavage des Abdomens) und verzichtet auf jegliche Anastomose oder Stomaanlage. Die Darmenden werden blind (in Klammernahttechnik) verschlossen. Danach folgt eine Phase der intensivmedizinischen Stabilisierung. Die endgültige Entscheidung bezüglich der Anastomosenanlage fällt dann erst im Rahmen eines zweitzeitigen Eingriffs ca. 24-48 h später. Auf diese Weise ist es möglich, die Allgemeinsituation des Patienten und seine Entwicklung besser $\mathrm{zu}$ beurteilen. Ein weiterer Vorteil ist die Tatsache, dass die unter Umständen sehr schwierige Entscheidung der Anastomosierung oder Diskontinuitätsoperation nicht unter dem Druck einer Notfallsituation getroffen werden muss. Erste Studien zeigen, dass mit dieser Vorgehensweise die Rate der Hartmannresektionen deutlich gesenkt werden kann.

Die laparoskopische Lavage ist eine zusätzliche Therapievariante in der Notfallversorgung der perforierten Divertikulitis, die intensiv diskutiert wird. Bei dieser Operationstechnik beschränkt man sich ausschließlich auf eine Lavage des Abdomens mit mehreren Litern und verzichtet auf jegliche Mobilisation, Präparation oder gar Resektion. Ein Problem bei der Beurteilung des Stellenwertes ist das zum Teil sehr inhomogene Patientengut: Unter der Definition einer perforierten Divertikulitis sind solche Patienten mit lokal begrenzter freier Luft ebenso zu verstehen, wie solche mit eitriger oder kotiger Peritonitis. Außerdem zeigen Studien, die explizit Patienten im Stadium Hinchey III (generalisierte eitrige Peritonitis = Typ 2c1) vergleichen, zum Teil massiv divergierende Ergebnisse: Die einen berichten über ein sehr gutes und geeignetes Instrument in der Notfallsituation, die anderen haben die Studie aufgrund erheblicher Komplikationen im laparoskopischen Lavage-Arm frühzeitig beendet. Zudem ist das Therapieziel nicht immer eindeutig zu erkennen: Manche betrachten die laparoskopische Lavage lediglich als sogenanntes „Bridging“-Verfahren, das geeignet ist die Rate einer Diskontinuitätsresektion und einer Stomaanlage zu senken. In dieser Variante erhalten allen Patienten dann einen elektiven oder auch frühselektiven resezierenden laparoskopischen Zweiteingriff. Andere sehen in der laparoskopischen Lavage (bei einer purulenten oder gar kotigen Peritonitis) ein Instrument der definitiven Therapie und beschränken sich im Weiteren lediglich auf eine Verlaufskontrolle. Zum aktuellen Zeitpunkt ist es daher nicht möglich, diese Methodik abschließend zu bewerten. Die Anwendung sollte weiterhin nur unter Studienbedingungen durchgeführt werden. 


\section{Weiterführende Literatur}

Di Saverio S, et al. The Ladies Trial: Premature termination of the LOLA arm and increased adverse events incidence after laparoscopic lavage may be influenced by inter-hospital and inter-operator variability? Take-home messages from a center with laparoscopic colorectal expertise. Int J Surg. 2016;36(Pt A):118-120.

Galbraith N, et al. Laparoscopic Lavage in the Management of Perforated Diverticulitis: a Contemporary Meta-analysis. J Gastrointest Surg. 2017;21(9):1491-1499.

Gregersen R, et al. Treatment of patients with acute colonic diverticulitis complicated by abscess formation: A systematic review. Int J Surg. 2016;35:201-208.

Leifeld L, et al. S2k guidelines diverticular disease/diverticulitis. Z Gastroenterol. 2014;52(7):663-710.

Sohn M, et al. Perforated Diverticulitis with Generalized Peritonitis: Low Stoma Rate Using a „Damage Control Strategy“. World J Surg. 2018;42(10):3189-3195.

Sohn M, et al. Damage control strategy for the treatment of perforated diverticulitis with generalized peritonitis. Tech Coloproctol. 2016;20(8):577-583.

Thornell A, et al. Laparoscopic Lavage for Perforated Diverticulitis With Purulent Peritonitis: A Randomized Trial. Ann Intern Med. 2016;164(3):137-145.

van de Wall B, Draaisma WA, van der Kaaij RT, et al. The value of inflammation markers and body temperature in acute diverticulitis. Colorectal Dis. 2013;15(5):621-626. doi: 10.1111/codi.12072. 
